\title{
Medidas de Acessibilidade a Oportunidades em Estudos Urbanos
}

\author{
Accessibility Measures to Opportunities in Urban Studies \\ Medidas de Accesibilidad a Oportunidades en Estudios Urbanos
}

Jefferson Hishiyama.

Ingeniero Civil, M.Sc(c). Transportes Urbanos.

Universidade de Brasília (UnB), Brasilia, Brasil.

jefferson.hishiyama@gmail.com

(i) https://orcid.org/0000-0001-9631-3287

Pastor Willy Gonzales Taco.

Ingeniero Civil, M.Sc. Transportes Urbanos. Dr. en Ingeniería de Transportes.

Universidade de Brasília (UnB), Brasilia, Brasil.

pwgtaco@gmail.com

(iD) https://orcid.org/0000-0002-2055-9114

Recibido: noviembre 23 de 2020

Aceptado: enero 5 de 2021

Publicado: enero 10 de 2021

\section{RESUMO}

Este estudo tem como objetivo identificar as abordagens de mensuração da acessibilidade, seus indicadores e principais temas de aplicação nos sistemas de uso do solo e transportes. Por meio de método híbrido de revisão sistemática da literatura e análise bibliométrica de redes de acoplamento bibliográfico, foram selecionadas 54 publicações e identificados 7 temas de aplicações (transporte público, sistemas de transporte, planejamento urbano, uso do solo, ambiente construído, análises econômicas e novas tecnologias). Verificou-se a versatilidade das medidas de acessibilidade em estudos urbanos e observou-se maior oportunidade de contribuição científica em temas emergentes relacionados a novas tecnologias e suas implicações no planejamento urbano e de transportes.

Palavras-chave: Acessibilidade; transporte; uso do solo; revisão sistemática; acoplamento bibliográfico.

\section{ABSTRACT}

This study aims to identify the approaches to measure accessibility, their indicators and main application themes in land use and transport systems. Through a hybrid method of systematic literature review and bibliometric analysis of bibliographic coupling networks, 54 publications were selected and 7 application themes were identified (public transport, transport systems, urban planning, land use, built environment, economic analysis and new technologies). The versatility of accessibility measures in urban studies was verified and there was a greater opportunity for scientific contribution in emerging themes related to new technologies and their implications for urban and transport planning.

Keywords: Accessibility; transport; land use; systematic review; bibliographic coupling. 


\section{RESUMEN}

Este estudio tiene como objetivo identificar los enfoques para medir la accesibilidad, sus indicadores y los principales temas de aplicación en el uso del suelo y los sistemas de transporte. Mediante un método híbrido de revisión sistemática de la literatura y análisis bibliométrico de redes de acoplamiento bibliográfico, se seleccionaron 54 publicaciones y se identificaron 7 temas de aplicación (transporte público, sistemas de transporte, planificación urbana, uso del suelo, entorno construido, análisis económico y nuevas tecnologías). Se verificó la versatilidad de las medidas de accesibilidad en los estudios urbanos y hubo una mayor oportunidad de contribución científica en temas emergentes relacionados con las nuevas tecnologías y sus implicaciones para la planificación urbana y del transporte.

Palabras clave: Accesibilidad; transporte; uso del suelo; revisión sistemática; acoplamiento bibliográfico.

\section{INTRODUÇÃO}

A acessibilidade representa uma importante característica urbana, estando diretamente relacionada ao planejamento de transporte e uso do solo. Uma das primeiras abordagens de mensuração da acessibilidade foi estabelecida por Hansen (1959), que a caracterizou como uma medida de distribuição espacial de um potencial de oportunidades (destinos) atingíveis através do sistema de transporte por pessoas ou instituições. Em seu estudo, o autor analisou que os desejos de viagens dos indivíduos e suas decisões de escolha residencial de uso do solo estão correlacionadas no contexto urbano. A partir de então, pesquisadores de distintas áreas do conhecimento procuram entender, integrar e prever a relação entre as políticas de uso do solo e os padrões de viagem por meio da acessibilidade (Acheampong \& Silva, 2015; Kii et al., 2016).

No âmbito do planejamento de transportes, Murray et al. (1998) apontam que os sistemas de transportes formam a base na qual o desenvolvimento econômico de uma região pode ocorrer e o meio pelo qual a interação da sociedade acontece. Assim, limitar acessibilidade significa limitar as oportunidades econômicas e sociais de determinada população.

Di et al. (2018) apontam que devido a transdisciplinaridade do planejamento de transportes, a mensuração da acessibilidade é frequentemente mensurada e conceituada de variadas perspectivas. Assim, a literatura ressalta que a acessibilidade carece de um método de mensuração e representação que seja passível de comparação em vários contextos, robusto e prático, necessitando de esforços de pesquisadores nesse âmbito (Acheampong \& Silva, 2015; Kii et al., 2016; Sousa et al., 2017; Di et al., 2018).

Nesse contexto, o artigo tem como objetivo identificar as abordagens de mensuração da acessibilidade, seus indicadores e principais temas de aplicação nos sistemas de transportes e uso do solo através de uma análise sistemática de literatura internacional. Serão utilizados como acervo as bases de dados Web of Science e Scopus, tendo como ferramentas de análise parâmetros bibliométricos.

Na sequência estão apresentados uma breve contextualização acerca das medidas de acessibilidade e técnicas de revisão sistemática da literatura e análise bibliométrica, a descrição do método adotado, os resultados encontrados, as discussões e considerações finais do estudo.

\section{MEDIDAS DE ACESSIBILIDADE}

As medidas de acessibilidade, segundo Geurs \& Van Wee (2004), possuem quatro componentes principais: ouso dosolo, otransporte, ofatortemporal e o fator individual. De acordo com os autores, o componente "uso do solo" está relacionado a oferta (quantidade, qualidade e distribuição espacial de oportunidades) e a demanda (população) para cada região. O componente "transporte" consiste no sistema utilizado pelo indivíduo para atender suas necessidades de deslocamento, sendo expresso em desutilidades, como tempo (de viagem, de espera e de estacionamento), custo e esforço (confiabilidade de serviço, conforto, risco, etc.). O componente "transporte" é dependente de sua infraestrutura, localização e características, como velocidade, capacidade e custos. Já o fator "tempo" 
considera a disponibilidade das oportunidades em diferentes horários e dias. Por sua vez, o fator "individual" reflete as necessidades (dependente da idade, renda, formação familiar, etc.), habilidades (condições físicas) e oportunidades (renda, custo de viagem, educação) individuais. Ainda, esses autores ressaltam que o fator "individual" afeta diretamente o nível de acesso da população aos meios de transporte e às oportunidades disponíveis, influenciando fortemente na mensuração agregada total de acessibilidade.

Dada a gama de variáveis que podem compor as medidas de acessibilidade, Handy \& Neimeier (1997) afirmam que não há uma melhor abordagem para sua mensuração, uma vez que diferentes situações e objetivos demandam diferentes abordagens. Nesse entendimento, Geurs \& Ritsema van Eck (2001) apontam três perspectivas para a mensuração da acessibilidade: i) medidas baseadas em infraestrutura; ii) medidas baseadas em atividades; e iii) medidas baseadas em utilidade.

As medidas baseadas em infraestrutura são vistas como medidas de performance, tendo como principais variáveis o tempo de viagem, congestionamento e velocidade de operação. Para Geurs \& Ritsema van Eck (2001) embora essa abordagem resulte em importantes análises de níveis de serviço, elas falham ao não identificar que destinos de interesse podem estar relativamente distantes do ponto avaliado.

Já as medidas baseadas em atividades avaliam a distribuição das oportunidades no espaço, podendo ser subclassificadas em quatro aproximações: medidas de distância, medidas de contorno, medidas de potencial e medidas derivadas da geografia tempo-espaço. As medidas de distância são consideradas medidas de "acessibilidade relativa", tendo como variáveis usualmente o tempo máximo de viagem ou a distância euclidiana ou a percorrida. As medidas de contorno consideram como variável o número de oportunidades que podem ser alcançadas dentro de uma restrição de tempo ou distância. Já as medidas de potencial são baseadas no modelo gravitacional e estimam a acessibilidade de todas as zonas considerando uma função de decaimento relacionada à atração com o aumento do custo (tempo, distância, entre outros). Por sua vez, as medidas derivadas da geografia tempo-espaço consideram a disponibilidade de atividades em distintos horários do dia e de vezes que um indivíduo pode participar de tais eventos, nessa medida o uso do solo e transporte apresentam igual importância.

As medidas de utilidade são baseadas na teoria econômica de utilidade, as quais entendem acessibilidade como um resultado de benefício para a escolha de transporte dado um grupo máximo de opções.

Outras perspectivas para mensuração podem ser encontradas na literatura, como, por exemplo, as de Handy \& Neimeier (1997) que classificam mensurações em medidas de oportunidade cumulativa, medidas baseadas em gravidade e baseadas na teoria da utilidade randômica. Entretanto, para este estudo, serão consideradas as perspectivas de Geurs \& Ritsema van Eck (2001) para a categorização das publicações analisadas.

\section{Revisão Sistemática da Literatura e Análise Bibliométrica}

Najmi et al. (2017) destacam que as abordagens quantitativas complementam as abordagens nãoquantitativas. Neste sentido, o presente estudo adotará um método híbrido de análise de literatura, empregando o processo metodológico de Revisão Sistemática da Literatura (RSL), apresentado por Kitchenham \& Charters (2007), e utilizando parâmetros bibliométricos e rede de acoplamento bibliográfico para análise de qualidade e síntese de resultados.

Segundo Kitchenham \& Charters (2007), a RSL consiste na adoção de um método científico, não enviesado e passível de reprodução, para identificação, avaliação e interpretação de aspectos ou questionamentos relevantes sobre uma pesquisa, tópico ou fenômeno de interesse. De modo geral, a RSL é utilizada por pesquisadores na busca por evidências científicas, estado do conhecimento e de possíveis lacunas presentes na literatura sobre um tema de interesse.

Entretanto, a Análise Bibliométrica ( $A B$ ) apresenta um panorama mais holístico da literatura, assim como a evolução e desenvolvimento do tema estudado (Najmi et al., 2017). Segundo Broadus (1987), estudos bibliométricos são caracterizados pela análise quantitativa de materiais publicados e suas características bibliográficas, utilizandose como parâmetros das publicações seus metadados de título, autores, afiliações, palavraschave, resumo, referências bibliográficas, 
citações e outros. A partir desses, são realizadas análises de rede, representados como mapas visuais de cocitações, coocorrência de autores e acoplamento bibliográfico. Nas redes de acoplamento bibliográfico os nós representam as publicações e suas dimensões indicam o número de citações, enquanto os arcos indicam a intensidade de proximidade entre duas ou mais publicações segundo as referências bibliográficas que são compartilhadas entre elas. Essa relação de indicadores bibliográficos permite que as publicações sejam organizadas em clusters temáticos, podendo ser rapidamente obtidos com o auxílio de ferramentas computacionais.

\section{MÉTODO}

Planejamento da Revisão. A primeira etapa se caracteriza pela definição da justificativa, problema e objetivos da pesquisa de modo a orientar a condução dos mecanismos de pesquisa e análise de resultados.

Condução da Revisão. Na segunda etapa são definidas as estratégias de pesquisa e suas limitantes por meio de cinco procedimentos: i) identificação da pesquisa; ii) seleção dos estudos primários; iii) análise de qualidade; iv) extração de dados; e, v) síntese dos dados. A seguir estão descritos os procedimentos adotados.

Identificação da pesquisa. No procedimento de "Identificação da pesquisa" é realizada a definição da estratégia de investigação e suas limitantes. De início, definiu-se que a busca seria realizada com horizonte temporal de publicações na íntegra até o mês de setembro de 2019, período no qual realizou-se o levantamento das publicações. Foram escolhidas as bases de dados Web of Science (WoS) e Scopus pela possibilidade de extração de dados bibliométricos dos resultados de busca. Quanto a identificação dos estudos, foram organizados três grupos de palavras chaves a serem identificadas tanto nos títulos dos documentos, quanto em seus resumos ou palavras chaves. As palavraschaves de pesquisa consideraram o arranjo entre os termos accessibility, model, modelling, measure, measurement e land use e/ou transport, transports e transportation. Foram excluídas as pesquisas relacionadas a acessibilidade física de pessoas com mobilidade reduzida com o uso do booleano NOT (WoS) e AND NOT (Scopus) em conjunto com a expressão disable.

Seleção dos estudos primários. Ainda na segunda etapa, foram selecionados nas bases de dados apenas trabalhos do tipo científicos, redigidos no idioma inglês e que pertencessem às áreas de estudo de engenharia de transportes, planejamento urbano e geografia, selecionando-se as áreas temáticas de Transportation, Geography e Urban Studies (WoS) Engineering e Social Science (Scopus). Ainda com critérios de seleção de publicações, foram utilizados os parâmetros bibliométricos de número médio de citações tidas pelo artigo por ano de publicação, sendo selecionados os artigos com média de 10 (dez) ou mais citações por ano. e identificador DOI.

Análise de qualidade. $O$ terceiro procedimento procura filtrar a aderência das publicações segundo os aspectos definidos na etapa 1 "Planejamento da revisão". Assim, foram selecionados os artigos que versassem sobre metodologias e abordagens de mensuração da acessibilidade para estudos de uso do solo e transporte por meio da leitura individual do conteúdo das publicações.

Extração de dados. Selecionadas as publicações relevantes, realizou-se o levantamento das informações relacionadas a abordagem de mensuração da acessibilidade, seus indicadores utilizados e temática para cada um dos estudos. Ressalta-se que a abordagem de mensuração foi classificada segundo as perspectivas propostas por Geurs \& Ritsema van Eck (2001).

Síntese dos dados. Caracteriza-se pela análise dos resultados de pesquisa, sendo realizada síntese descritiva dos resultados com uso de redes de acoplamento bibliográfico geradas com o auxílio do software VOSviewer. Ressalta-se que no presente estudo não foi realizada meta-análise com a utilização de procedimentos estatísticos na análise de resultados quantitativos de distintos estudos.

Elaboração do relatório de revisão. Consiste na apresentação dos resultados através da redação de um artigo de contribuição científica, apresentando os resultados e discussões. 


\section{RESULTADOS E DISCUSSÕES}

Análise dos metadados das bases de publicação. $\mathrm{Na}$ Tabela 1 apresenta-se o quantitativo de publicações existentes nas bases avaliadas (WoS e Scopus) em setembro de 2019 para cada um dos três grupos de palavras-chaves pesquisados. Observa-se que a base de pesquisa Scopus apresentou $60,13 \%$ publicações a mais que a busca realizada na Web of Science. Ambas as bases apresentaram maior número de resultados para o G2 (62,66\% dos resultados), sugerindo que existem mais modelos de mensuração envolvendo o componente "transporte" que o componente "uso do solo" na área temática de estudos da geografia urbana. Quanto ao G3, nota-se que embora a acessibilidade seja caracterizada como elemento da interação entre ambos os sistemas de uso do solo e transporte, os resultados mostram que, em volume de publicações, apenas $12,67 \%$ dos artigos extraídos contém simultaneamente ambos os termos.

Tabela 1. Total de publicações por grupos de palavraschave e base de dados.

\begin{tabular}{|c|c|c|c|}
\hline Grupo & String de pesquisa & $\begin{array}{l}\text { Web of } \\
\text { Science }\end{array}$ & Scopus \\
\hline G1 & $\begin{array}{l}\text { "accessibility" AND "land use" } \\
\text { AND ("model*" OR" measur*") }\end{array}$ & 600 & 895 \\
\hline G2 & $\begin{array}{l}\text { NOT "disaable*" } \\
\text { "accessibility "AND "trans- } \\
\text { port*" AND ("model*" OR" } \\
\text { measur*").NOT "disable*" }\end{array}$ & 1.316 & 2.482 \\
\hline G3 & $\begin{array}{c}\text { "accessibility" AND "land } \\
\text { use" AND "transport*" AND } \\
\text { ("model*" OR" measur*") NOT } \\
\text { "disable*" }\end{array}$ & 414 & 354 \\
\hline Total & & 2.330 & 3.731 \\
\hline
\end{tabular}

Quanto a evolução das publicações ao decorrer dos anos, a Figura 1 apresenta a curva de crescimento para cada um dos três grupos de palavras-chave e base de dados. Os resultados abrangeram uma janela temporal de 60 anos, sendo as publicações mais antigas de 1959 de Hansen e Hamburg \& Creighton, identificadas pelo grupo "G1 - Scopus". Nota-se que embora o grupo "G2 - Scopus" seja o mais volumoso em termos de publicações, essas estão presentes na base de dados apenas a partir de 1965. Observa-se ainda uma tendência acentuada de crescimento do número de publicações a partir do ano de 2007. De fato, ao analisar de modo segregado a proporção de publicações para cada um dos grupos em cada uma das bases, constata- se que o período entre 2007 a 2019 concentra mais de $90 \%$ da produção acadêmica identificada na WoS e mais de $80 \%$ na base Scopus.

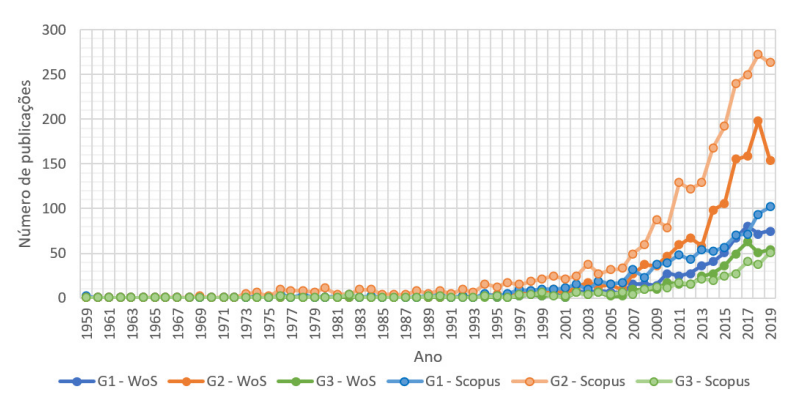

Figura 1. Evolução de publicações por ano Fonte: Autores (2020).

No âmbito da origem das publicações, os Estados Unidos apresentaram a maior participação, tendo uma proporção máxima de 27,6\% publicações no "G1 - WoS" (224) e "G2 - Wos" (152) e proporção mínima de 23,8\% no "G2 - Scopus" (735). Na sequência está a China, com aproximadamente 10\% de publicações, seguida do Reino Unido, Países Baixos, Canadá e Austrália, com média de publicações ao redor de 6\% cada. Destaca-se a carência de estudos internacionais relacionados ao Sul Global, como América Latina, África e Sudeste Asiático, cujos contextos históricos de uso do solo e do planejamento de transportes se diferem da realidade de países desenvolvidos.

Quanto aos veículos de publicação, os cinco principais periódicos e seus H-Index foram: Journal of Transport Geography (H-Index 83), Transportation Research Record (H-Index 93), Transportation Research Part A: Policy and Practice (H-Index 110), Transport Policy (H-Index 77) e Transportation (H-Index 70).

Rede de acoplamento bibliográfico e síntese descritiva. As buscas dos três grupos de pesquisa nas bases de dados resultaram no levantamento de 6.061 publicações, as quais, após exclusão de publicações repetidas e sem identificador DOI, obteve-se um total de 2.816 publicações para análise. No entanto, dado o grande volume de publicações, foi adotado o parâmetro bibliométrico de citações para a identificação dos trabalhos de maior relevância entre seus pares. Nesse sentido, foi considerada a média de citações por ano de publicação para cada um dos 2.816 estudos e 
então excluídos os trabalhos com média inferior a 10 citações por ano de publicação, sendo selecionados 109 estudos primários.

A partir do levantamento desses estudos, foi realizado o procedimento de análise de qualidade das publicações, que, resultou na seleção de 54 trabalhos, dos quais foram extraídas as informações quanto as abordagens de mensuração da acessibilidade utilizadas, seus indicadores e temática do estudo. Ainda foi realizada a análise de redes segundo acoplamento bibliográfico organizando os estudos em clusters segundo suas relações de compartilhamento de referências bibliográficas (conforme observado na Figura 2), o acoplamento bibliográfico agrupou os estudos selecionados em 7 clusters. Nos itens a seguir, será realizada uma breve síntese descritiva dos resultados encontrados para cada um dos clusters quanto aos aspectos de abordagens de mensuração da acessibilidade, indicadores e contexto de estudo.

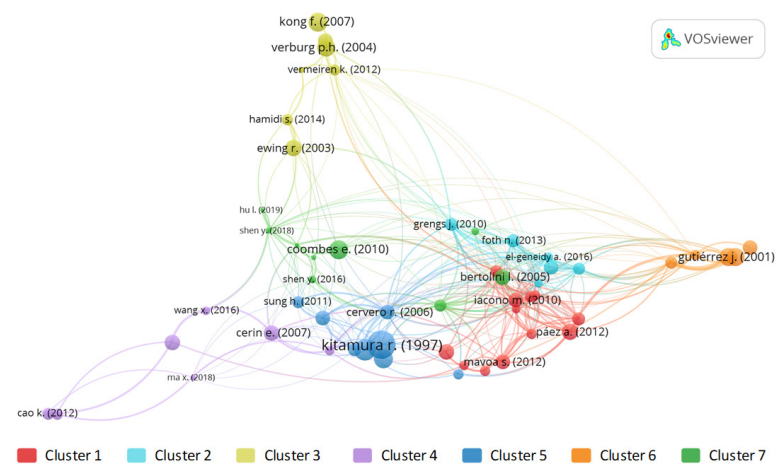

Figura 2. Rede de acoplamento bibliográfico.

Fonte: Autores (2020).

Cluster 1: Transporte público coletivo. O Cluster 1 é composto por 12 publicações, sendo o trabalho de Páez, Scott \& Morency (2012) o de maior número de citações (192). A principal temática de estudo do cluster está relacionada ao transporte público coletivo, presente em 8 trabalhos, na sequência destaca-se a temática de equidade (3 estudos), seguida de caminhabilidade (2 estudos), análise sociodemográfica (2 estudos) e veículo individual motorizado ( 2 estudos).

As principais abordagens de mensuração da acessibilidade utilizadas são medidas de contorno baseada em atividade e medidas de infraestrutura, cada uma presente em 4 estudos. Destaca-se ainda a utilização de medidas de distância baseadas em atividades (3), medidas de espaço-tempo baseadas em atividades (2) e medidas de potencial (gravidade) baseadas em atividade (2).

O principal indicador utilizado no cluster é o tempo de viagem (6), seguido da distância (5) e número de viagens (2). Foram identificados ainda indicadores de número de empregos, frequência de transporte público, número de locais de alimentação, creche, saúde e compras.

\section{Cluster 2: Sistemas de transporte e} oportunidades de emprego. O Cluster 2 é composto por 6 publicações, sendo o trabalho de Currie (2010) o de maior número de citações (162). Assim, como o Cluster 1, o principal tema de estudo está relacionado ao transporte público (5 estudos), seguido da equidade (2 estudos) e planejamento tarifário, planejamento de transportes e network design. Esse aspecto pode indicar a proximidade entre os dois grupos na rede de acoplamento bibliográfico, como observado na Figura 2.

Diferentemente do Cluster 1, a principal abordagem de mensuração da acessibilidade desse grupo é de medidas de potenciais (gravidade) baseadas em atividade (3), seguida de medidas de espaçotempo baseadas em atividade (2) e de medidas de utilidade (2).

Por sua vez, o principal indicador utilizado por esse grupo é número de empregos (4), sendo utilizado também tempo de viagem (1), distância (1) e número de viagens (1).

Cluster 3: Planejamento urbano. O Cluster 3 é composto por 7 estudos, sendo o trabalho de Verburg, Ritsema van Eck \& Nijs (2004) o de maior número de citações (222). O principal tema de estudo abordado pelo grupo é o planejamento urbano ( 5 estudos), seguido da análise de dispersão urbana ( 3 estudos), uso do solo ( 2 estudos) e house pricing (1 estudo).

A principal abordagem de mensuração da acessibilidade utilizada é a de medidas de infraestrutura (4), seguido das medidas de distância baseada em atividades (2) e medidas de potencial (gravidade) baseada em atividade (1).

Os principais indicadores utilizados pelo grupo é o tempo de viagem (3) e distância (3), sendo também adotados indicadores como comprimento de vias (1), área construída (1) e densidade de vias (1). 
Destaca-se que nesse cluster o estudo de Hadimi \& Ewing (2014) adotou uma abordagem de mensuração mista entre infraestrutura e uso do solo, considerando como indicador de acessibilidade a densidade de vias baseada na dimensão das quadras e tipologias de interseções.

Essas características relacionadas à temática de estudo e indicadores utilizados pelo cluster acabam por posicioná-lo relativamente distante dos demais clusters na rede de acoplamento bibliográfico (Figura 2).

Cluster 4: Uso do solo. O Cluster 4 é composto por 7 estudos, sendo o trabalho de Cerin et al. (2007) o de maior número de citações (175). A temática preponderante é a otimização do uso do solo (4 estudos) e seu impacto na acessibilidade local a infraestrutura de transporte, tendo outros temas como Desenvolvimento Orientado ao Transporte - DOT (2 estudos), bike sharing (2 estudos) e caminhabilidade (1 estudo).

Entre as abordagens de mensuração da acessibilidade nota-se a utilização de medidas de distância baseadas em atividade (2), assim como a utilização de medidas de infraestrutura (1), medidas de potencial (gravitacional) e de contorno (1).

Já os principais indicadores adotados pelo Cluster 4 são tempo de viagem (2) e número de empregos (2), sendo também considerados número de viagens (1), número de pontos de parada (1), número de linhas (1) e volume de tráfego (1).

Ressalta-se que os trabalhos de Cao et al. (2011) e Cao et al. (2012) não estão contabilizados nas abordagens e indicadores mencionadas anteriormente por utilizarem a mensuração da acessibilidade através de uso do solo, tendo como indicador a hierarquia viária. Pode-se observar pela Figura 2 que esses dois trabalhos se localizam distante dos demais trabalhos selecionados.

\section{Cluster 5: Ambiente construído e oportunidades} urbanas. O Cluster 5 é composto por 8 estudos, sendo o trabalho de Kitamura, Mokhtarian \& Laidet (1997) o de maior número de citações (562). Os aspectos relacionados ao tema de planejamento urbano são os mais presentes no grupo (6 estudos). Em conjunto, estão os temas de uso do solo (3 estudos), transporte público (2 estudos), meio ambiente (1 estudo), equidade (1 estudo) e realocação residencial (1 estudo). De modo geral, o cluster aborda aspectos relacionados ao ambiente urbano construído.

Diferentemente do Cluster 4, as análises acerca do planejamento urbano são abordadas principalmente a partir de medidas de potencial (gravidade) baseada em atividades (3) e de contorno baseadas em atividades (3), sendo também adotadas medidas de utilidade (1) e medidas de distância baseada em atividade (1).

Tem-se como principais indicadores utilizados pelo Cluster 5 o número de empregos (4), o número de estabelecimentos comerciais (3), a distância (2) e o tempo de viagem (2). Entretanto, foram também utilizados o número de equipamentos de saúde, a população, o número de pontos de ônibus, a densidade de habitações e área de quarteirões.

Cluster 6: Análises econômicas de investimento em infraestruturas. O Cluster 6 é composto por 5 estudos, sendo o trabalhos de Gutiérrez (2001) e Vickerman, Spiekermann \& Wegener (1999) os com maior número de citações, 234 cada. A principal temática de pesquisa do grupo são análises econômicas relacionadas a investimentos em infraestrutura de transporte, tendo como principais temáticas redes de transporte (3 estudos), trens de alta velocidade (2) e avaliação de desastres (1).

Entre as abordagens para mensuração da acessibilidade, identificaram-se medidas de potencial (gravidade) baseadas em atividade (5), seguida de medidas de infraestrutura (3) e medidas de contorno baseadas em atividade (2).

Dada a característica de análise econômica de infraestruturas, os principais indicadores são tempo de viagem (4), produto interno bruto (PIB) (3) e população (3), sendo também utilizados número de destinos (1), distância (1) e volume de tráfego (1).

\section{Cluster 7: Temas emergentes e novas tecnologias.} O Cluster 7 é composto por 9 estudos, sendo o trabalho de Coombes, Jones \& Hillsdon (2010) o de maior número de citações (259). Esse cluster possui duas principais características, primeiro, é o grupo que apresenta a maior quantidade de trabalhos recentes ( 6 das 9 publicações são dos últimos 5 anos), e segundo, apresenta uma grande dispersão (Figura 2). Essas características fazem com que o cluster tenha como temática estudos emergentes e novas tecnologias que orbitam estudos relacionados ao planejamento de transporte e ao 
uso do solo. Os temas abordados são a utilização de data science (2) transporte público urbano (2 estudos), caminhabilidade (2 estudos), equidade (2 estudos), saúde (1 estudo), uso do espaço público (1 estudo), house pricing (1 estudo), dockless bike sharing (1 estudo) e veículos autônomos (1 estudo).

As principais abordagens de mensuração da acessibilidade são medidas de distância baseadas em atividade (4), medidas de infraestrutura (3), medidas de contorno (2), medidas de potencial (gravitacional) baseadas em atividades (1) e medidas de utilidade (1).

Por sua vez, os principais indicadores utilizados são a distância (4) e o tempo de viagem (2). Entretanto, também são utilizados o número de empregos, a frequência das linhas de ônibus, o número de estações e pontos de embarque, o número de oportunidades, a densidade de vias e diversidade de uso do solo.

Destaca-se que o trabalho de Su et al. (2019) não está considerado nas contagens anteriores pois utiliza como abordagem de mensuração da acessibilidade parâmetros de uso do solo, como índice de entropia e índice de diversidade de uso do solo de Simpson, que não permitem uma classificação dentro das perspectivas de Geurs \& Ritsema van Eck (2001).

\section{CONSIDERAÇÕES FINAIS}

O presente estudo propôs identificar um panorama geral sobre as abordagens de mensuração da acessibilidade, assim como seus indicadores e principais temas de aplicação nos sistemas urbanos de uso do solo e transportes. Por meio de uma abordagem híbrida de Revisão Sistemática da Literatura (RSL) com análise bibliométrica de redes de acoplamento bibliográfico, foram identificadas 7 principais áreas de estudo segundo características intrínsecas das publicações que as compõem, sendo elas relativas ao transporte público, sistemas de transporte, planejamento urbano, uso do solo, ambiente construído, análises de avaliação econômica e novas tecnologias.

Através da síntese descritiva e da análise da rede de acoplamento bibliográfico, observou-se que trabalhos que compartilham indicadores e abordagens de mensuração semelhante tendem a estar menos dispersos, o que acaba resultando em um certo consenso quanto a melhor abordagem mais sólida a ser utilizada em determinadas situações. Nesse âmbito, destaca-se, por exemplo - Cluster 6, cuja temática orbita em avaliação econômica de infraestrutura de transportes e os estudos utilizam, em sua maioria, medidas de potencial baseado em atividades derivados do modelo gravitacional, tendo como indicadores da função de decaimento o PIB de determinada área.

Ainda se observa que a avaliação de acessibilidade em transporte público segue duas frentes de estudo, a primeira (Cluster 1 ) tem uma abordagem a partir de indicadores de medidas relativas, como tempo de viagem e distância, enquanto a segunda (Cluster 2) utiliza majoritariamente medidas baseadas no número de empregos disponíveis a determinada população.

Os estudos relacionados ao planejamento de transporte e otimização do uso do solo também se apresentaram em 3 grupos distintos pelo contexto de estudo. Os trabalhos relacionados ao estudo da dispersão urbana no planejamento urbano (Cluster 3) tendem a utilizar medidas de infraestrutura. Por sua vez, estudos mais emergentes, como a análise da otimização do solo para aumento da acessibilidade local motivada pela infraestrutura de transporte (Cluster 4) carece de consenso de abordagem e indicadores, sendo observada a existência de outras medidas de mensuração, como a hierarquia viária, por exemplo. Já um terceiro grupo (Cluster 5) avalia a influência dos sistemas de transporte na localização de atividades no espaço urbano, como empregos e comércio, utilizando, por tanto, principalmente medidas baseadas em atividade.

Observa-se ainda que temas emergentes com pesquisas publicadas nos últimos 5 anos, como, por exemplo, data science, dockless bike sharing e veículos autônomos, ainda se apresentam como estudos de caráter exploratório, onde avaliam-se distintas medidas e abordagens de mensuração da acessibilidade segundo o contexto de estudo, carecendo de uma uniformidade de abordagens e indicadores utilizados para avaliar o espaço urbano.

De modo geral, observou-se que as formas de abordagem de mensuração da acessibilidade e os indicadores que são utilizados variam conforme a temática de estudo, evidenciado a argumentação de Handy \& Neimeier (1997) que já ressaltavam a falta de uma melhor abordagem para a mensurar 
a acessibilidade, visto que diferentes situações e objetivos demandam diferentes abordagens. No entanto, destaca-se que alguns temas apresentam maior robustez na mensuração da acessibilidade, como avaliação econômica e transporte público, entretanto outras áreas necessitam de maior contribuição científica, em especial os assuntos emergentes relacionados a novas tecnologias e suas implicações no planejamento urbano de uso do solo e de transportes.

Finalmente, ressalta-se, queesse trabalhoapresenta restrições. Foram analisados apenas trabalhos publicados na língua inglesa, desconsiderando potenciais contribuições que possam existir em outros idiomas, e ainda, a busca de dados foi realizada apenas nas bases Web of Science e Scopus que, até o conhecimento dos autores, são as únicas bases que permitem extração automática de dados bibliométricos de pesquisa.

\section{AGRADECIMENTOS}

Os autores agradecem o Grupo de Pesquisa Comportamento em Transportes e Novas Tecnologias (CTNT) do Programa de PósGraduação em Transportes (PPGT) da Universidade de Brasília (UnB) pelos incentivos e apoio recebidos para o desenvolvimento deste trabalho.

\section{REFERÊNCIAS}

Acheampong, R. A., \& Silva, E. A. (2015). Land use-transport interaction modeling: A review of the literature and future research directions. Journal of Transport and Land Use, 8(3), 11-38. DOI: https://doi. org/10.5198/jtlu.2015.806

Broadus, R. N. (1987). Toward a definition of "bibliometrics". Scientometrics, 12(5-6), 373-379. DOI: https:// doi.org/10.1007/BF02016680

Cao, K., Batty, M., Huang, B., Liu, Y., Yu, L., \& Chen, J. (2011). Spatial multi-objective land use optimization: Extensions to the non-dominated sorting genetic algorithm-II. International Journal of Geographical Information Science, 25(12), 1949-1969. DOI: https://doi.org/10.1080/13658816.2011.570269

Cao, K., Huang, B., Wang, S., \& Lin, H. (2012). Sustainable land use optimization using Boundary-based Fast Genetic Algorithm. Computers, Environment and Urban Systems, 36(3), 257-269. DOI: https://doi. org/10.1016/j.compenvurbsys.2011.08.001

Cerin, E., Leslie, E., Toit, L. du, Owen, N., \& Frank, L. D. (2007). Destinations that matter: Associations with walking for transport. Health and Place, 13(3), 713-724. DOI: https://doi.org/10.1016/j.healthplace.2006.11.002

Coombes, E., Jones, A. P., \& Hillsdon, M. (2010). The relationship of physical activity and overweight to objectively measured green space accessibility and use. Social Science and Medicine, 70(6), 816-822. DOI: https://doi.org/10.1016/j.socscimed.2009.11.020

Currie, G. (2010). Quantifying spatial gaps in public transport supply based on social needs. Journal of Transport Geography, 18(1), 31-41. DOI: https://doi.org/10.1016/j.jtrangeo.2008.12.002

Di, Z., Yang, L., Qi, J., \& Gao, Z. (2018). Transportation network design for maximizing flow-based accessibility. Transportation Research Part B: Methodological, 110, 209-238. DOI: https://doi.org/10.1016/j. trb.2018.02.013

Geurs, K., \& Ritsema van Eck, J. R. (2001). Accessibility measures: review and applications. RIVM. Urban Research Centre, Utrecht University. 
Geurs, K., \& van Wee, B. (2004). Accessibility evaluation of land-use and transport strategies: Review and research directions. Journal of Transport Geography, 12(2), 127-140. DOI: https://doi.org/10.1016/j. jtrangeo.2003.10.005

Gutiérrez, J. (2001). Location, economic potential and daily accessibility: An analysis of the accessibility impact of the high-speed line Madrid-Barcelona-French border. Journal of Transport Geography, 9(4), 229-242. DOI: https://doi.org/10.1016/S0966-6923(01)00017-5

Hamidi, S., \& Ewing, R. (2014). A longitudinal study of changes in urban sprawl between 2000 and 2010 in the United States. Landscape and Urban Planning, 128, 72-82. DOI: https://doi.org/10.1016/j. landurbplan.2014.04.021

Handy, S. L., \& Niemeier, D. A. (1997). Measuring accessibility: An exploration of issues and alternatives. Environment and Planning A, 29(7), 1175-1194. DOI: https://doi.org/10.1068/a291175

Hansen, W. G. (1959). How Accessibility Shapes Land Use. Journal of the American Planning Association, 25(2), 73-76. DOI: https://doi.org/10.1080/01944365908978307

Kii, M., Nakanishi, H., Nakamura, K., \& Doi, K. (2016). Transportation and spatial development: An overview and a future direction. Transport Policy, 49, 148-158. DOI: https://doi.org/10.1016/j.tranpol.2016.04.015

Kitamura, R., \& Mokhtarian, P. L. (1997). A micro-analysis of land use and travel in five neighborhoods in the San Impacts of travel-based multitasking on mode choice and value of time View project Travel satisfaction View project. Transportation, 24, 125-158. DOI: https://doi.org/10.1023/A:1017959825565

Kitchenham, B., \& Charters, S. (2007). Guidelines for performing Systematic Literature Reviews in Software Engineering. In EBSE: Vol, 2.3.

Murray, A. T., Davis, R., Stimson, R. J., \& Ferreira, L. (1998). Public transportation access. Transportation Research Part D: Transport and Environment, 3(5), 319-328. DOI: https://doi.org/10.1016/S1361-9209(98)00010-8

Najmi, A., Rashidi, T. H., Abbasi, A., \& Travis Waller, S. (2017). Reviewing the transport domain: an evolutionary bibliometrics and network analysis. Scientometrics, 110(2), 843-865. DOI: https://doi.org/10.1007/ s11192-016-2171-3

Páez, A., Scott, D. M., \& Morency, C. (2012). Measuring accessibility: Positive and normative implementations of various accessibility indicators. Journal of Transport Geography, 25, 141-153. DOI: https://doi. org/10.1016/j.jtrangeo.2012.03.016

Sousa, F. F. L. de M., Loureiro, C. F. G., \& Lopes, A. S. (2017). Representação do fenômeno urbano por meio de modelos integrados dos transportes e uso do solo: revisão da literatura e discussão conceitual. Transportes, 25(4), 96. DOI: https://doi.org/10.14295/transportes.v25i4.1319

Su, S., Zhou, H., Xu, M., Ru, H., Wang, W., \& Weng, M. (2019). Auditing street walkability and associated social inequalities for planning implications. Journal of Transport Geography, 74(129), 62-76. DOI: https://doi. org/10.1016/j.jtrangeo.2018.11.003

Verburg, P. H., Ritsema van Eck, J. R., de Nijs, T. C. M., Dijst, M. J., \& Schot, P. (2004). Determinants of land-use change patterns in the Netherlands. Environment and Planning B: Planning and Design, 31(1), 125-150. DOI: https://doi.org/10.1068/b307

Vickerman, R., Spiekermann, K., \& Wegener, M. (1999). Accessibility and economic development in Europe. Regional Studies, 33(1), 1-15. DOI: https://doi.org/10.1080/00343409950118878 